
\title{
3 Research Square \\ PET/MR Imaging for the Evaluation of Cervical Cancer During Pregnancy
}

\section{Tatsuya Ishiguro ( $\nabla$ tishigur@med.niigata-u.ac.jp )}

Niigata University Medical and Dental Hospital https://orcid.org/0000-0002-6445-4066

\section{Nobumichi Nishikawa}

Niigata university

\section{Shiro Ishii}

Fukushima Medical University Hospital

\section{Kosuke Yoshihara}

Niigata University

Kazufumi Haino

Niigata university

Masayuki Yamaguchi

Niigata university

\section{Sosuke Adachi}

Niigata University: Niigata Daigaku

\section{Takafumi Watanabe}

Fukushima medical university hospital

\section{Shu Soeda}

Fukushima medical university hospital

\section{Takayuki Enomoto}

Niigata university

\section{Research article}

Keywords: cervical cancer, pregnancy, PET-MRI, metastasis, radical trachelectomy

Posted Date: October 5th, 2020

DOl: https://doi.org/10.21203/rs.3.rs-37453/v1

License: (1) This work is licensed under a Creative Commons Attribution 4.0 International License. Read Full License 
Version of Record: A version of this preprint was published at BMC Pregnancy and Childbirth on April 10th, 2021. See the published version at https://doi.org/10.1186/s12884-021-03766-w. 


\section{Abstract}

Background: To assess the diagnostic value of positron emission tomography/magnetic resonance imaging (PET/MRI) for cervical cancer propagation before surgery, especially in pregnant women.

Methods: 18F Fluorodeoxyglucose (FDG)-PET/MRI was performed in seven pregnant cervical cancer patients (28 - 34 years old) at 9-18 gestational weeks. In case \#5, a second PET/MRI was performed at 24 gestational weeks.

Results: Of seven FDG-PET/MRI examination series in six cases (cases \#1-\#6), FDG-PET/MR imaging could detect cervical tumors with abnormal FDG accumulation; these tumors were confirmed with a standardized uptake value max (SUV max) titer of 4.5 - 16. A second PET/MRI examination in case \#5 revealed the same SUV max titer as the first examination. In these six imaging series (cases \#1-\#5), there were no signs of cancer metastasis to the parametrium and lymph nodes. However, in case \#6, abnormal FDG accumulation in the left parametrial lymph nodes was also detectable. Pathological examination showed lymph node metastasis in case \#6. In case \#7, PET/MRI could not detect any abnormal FDG accumulation in the cervix and other sites. Cone biopsy demonstrated only micro-invasive squamous cell carcinoma. After treatment for cervical cancer, all seven patients have had no recurrence of disease within the follow-up period ( $2.4-5.2$ years), and their children have developed appropriately.

Conclusion: PET/MRI is an effective and relatively safe imaging tool to evaluate cervical cancer progression in pregnancy.

\section{Background}

Uterine cervical cancer is one of the most common gynecological cancers. Since most cervical cancers develop as a result of persistent high-risk human papilloma virus (HPV) infection and multi-step carcinogenesis ${ }^{1,2}, \mathrm{HPV}$ vaccination and regular cancer screening, including HPV-DNA tests and Pap smears, are effective for the prevention of invasive cervical cancer in many women. Nevertheless, there are serious concerns related to cervical cancer in Japan. Firstly, the rate of the HPV vaccination of young women has dropped from $70 \%$ in 2013 to less than 1\% in 2015 due to the Japanese Government's suspension of proactive recommendations for the HPV vaccine due to suspected adverse events after HPV vaccination ${ }^{3}$. Secondly, the rate of women, especially young women, who undergo cervical cancer screening is extremely low compared to that in other developed countries ${ }^{4}$. Additionally, many younger women undergo a cervical cancer screening examination only at the initial prenatal examination in Japan. As a result, the disease rate of cervical cancer in Japan (13.3/100,000 women) is about the same as that in low- and middle-income countries (15.7/100,000 women) and higher than that in high-income countries $(9.9 / 100,000 \text { women })^{1,5}$.

Recently, malignancy during pregnancy has increased and the most common type of malignancy is uterine cervical cancer ${ }^{6,7}$. Cervical cancer during pregnancy is mostly stage I disease, and the standard 
care of the International Federation of Gynecology and Obstetrics (FIGO) stage IB1 cervical cancer in pregnancy is radical hysterectomy with fetus in utero or cesarean radical hysterectomy ${ }^{7}$. Although it is most important to save maternal lives, patients occasionally wish to continue their pregnancy. To achieve the wish of patients without severe adverse effects on the fetus, we have previously reported the usefulness of abdominal radical tracherectomy during pregnancy (ART-DP) for stage IB1 cervical cancer 8 . Since pregnancy does not have a negative effect on cervical cancer prognosis, careful clinical and radiological follow up is another permissible treatment option for stage IB1 cervical cancer during pregnancy ${ }^{7}$.

To plan the treatment, it is important to look for signs of metastasis before surgery. Cervical cancer progresses directly into the parametrium, vagina, uterus, and adjacent organs. In addition, it may progress further by spreading to the regional lymph nodes. Lymph node metastasis is one of the important risk factors of disease progression ${ }^{9}$. The incidence rate of pelvic lymph node metastasis with T-stage IA1, IA2, IB1, and IIA1 was reported as $1 \%, 0-4.8 \%, 13.9 \%$, and $38.1 \%$, respectively $10-12$, and the rate of paraaortic lymph node metastasis with T-stage IB1 was $2-4 \%{ }^{13}$. Therefore, the latest staging system of FIGO includes the lymph node metastasis status; cases with lymph node metastasis are diagnosed as stage IIIC ${ }^{14}$.

To detect metastasis to the lymph node and parametrium before surgery, contrast-enhanced imaging including CT and MR are useful methods. However, the use of contrast-enhanced imaging during pregnancy, especially early gestation, must be avoided so as not to harm the fetus. Recently, fluorodeoxyglucose-positron emission tomography (FDG-PET) has been used to detect metastatic lesions of many types of cancer ${ }^{15}$. FDG-PET combined with CT or MRI has higher sensitivity than usual contrastenhanced imaging for malignant tumors ${ }^{16}$. In this article we discuss the usefulness of FDG-PET/MRI for cervical cancer treatment planning during pregnancy in seven cases of pregnant patients diagnosed with cervical cancer.

\section{Methods}

\section{Cases}

A total of seven pregnant patients with cervical cancer were enrolled in this exploratory case series. They were treated at the Niigata Medical and Dental Hospital, Niigata, Japan between 2013 and 2020. Abnormal cervical cells of all cases were first detected in early gestation by Pap smear screening, and malignant cervical tissues were subsequently confirmed by preoperative histological examination. The information related to these seven cases is summarized in Table 1. Of the seven cases presented in this study, three cases (cases \#1-\#3) have been previously published ${ }^{8}$.

\section{F-FDG-PET/MRI}


PET/MRI data were acquired on an integrated PET/MRI system (Biograph mMR; Siemens Healthcare) with a 3.0-Tesla MRI. All patients fasted for at least 4 hours or skipped one meal before the examination. The patients were injected with $4 \mathrm{MBq} / \mathrm{kg}$ of FDG, and PET/MR acquisition was started approximately one hour after the FDG injection. The mean glucose level at the time of injection was $83.5 \pm 6.5 \mathrm{mg} / \mathrm{dl}$ (range $=78-97$ ). No contrast agent was used in any of the patients.

Acquisition of the PET data started from the upper thigh, progressing upwards to the head, under shallow breathing. MRI was acquired simultaneously with PET acquisition. The PET data were acquired for 4-6 bed positions with 3 min per bed position for the whole-body, followed by 10 min for the pelvis with a matrix of $172 \times 172$, and reconstructed using OSEM 3D.

Axial T2WI-HASTE (half-Fourier acquisition single-shot turbo spin echo), coronal T1WI (Turbo spin echo coronal T1-weighted images) from the top of the head to the upper thigh, and lung volumetric interpolated breath-hold examinations (VIBE) were obtained for the screening of metastases. In addition, 3-mm thickness of sagittal and axial T2WI (turbo spin echo T2WI) and T1-weighted images, axial DWI, and 3-mm thickness of pelvic MRI were obtained for evaluation of the invasion of cervical cancer.

PET/MRI was performed at the Fukushima Medical University Hospital.

\section{Results}

FDG-PET/MR imaging was performed for seven pregnant patients (28 - 34 years old) at 9-18 gestational weeks in order to assess cervical cancer and metastatic lesions, including regional lymph nodes and the parametrium (Table 1). FDG-PET/MR imaging could detect cervical cancer with abnormal FDG accumulation in six FDG-PET/MRI examination series of the first five cases (cases \#1-\#5); these tumors were confirmed with a standardized uptake value max (SUV max) titer of 4.5 - 10 (Fig. 1). In case \#5, because uterine fibroid obstructed the ART-DP, FDG-PET/MRI was performed again at 24 gestational weeks to evaluate whether the patient could continue her pregnancy without any medical treatment for cervical cancer. The second FDG-PET/MRI examination showed the enhanced cervical tumor at the same SUV max titer as the first examination. In these six imaging series (cases \#1 - \#5), there were no signs of cancer metastasis to the parametrium and lymph nodes., In case \#6, however, abnormal signaling of the left parametrial lymph nodes with an SUV max of 8 was found. This signaling was accompanied by FDG accumulation in the cervical tumor (SUV max of 16). However, MR imaging without enhanced contrast could not definitively detect the lymph node metastasis (Fig. 2).

The first surgery, including pelvic lymphadenectomy, was performed 8 days to 7 weeks after FDG$\mathrm{PET} / \mathrm{MRI}$ examination. More than 15 lymph nodes were removed during the surgery in all six cases (cases \#1-\#6). Pathological examination showed the final diagnoses of squamous cell carcinoma (cases \#1, \#3, \#4, \#5, and \#7), adenocarcinoma (case \#2), and adenosquamous carcinoma (case \#6). In addition, surgical exploration found no evidence of metastasis in the five cases that were found to be without metastatic lesion by FDG-PET/MRI (cases \#1-\#5); in case \#6 where lymph node metastasis was detected by FDG-PET/MRI, pathological examination also mainly revealed left obturator and parametrial 
lymph nodes metastases of squamous cell carcinoma. In case \#7, the first punch biopsy sample of the uterine cervix revealed invasive carcinoma. Otherwise, PET/MRI could not detect any abnormal accumulation in the cervix or other sites. Cone biopsy demonstrated only micro-invasive squamous cell carcinoma with 2-mm deep stromal invasion.

Cesarean section after ART-DP was performed at 33 or 37 gestational weeks (cases \#1 - \#4). Simple hysterectomy was also performed at the same time as cesarean section; pathological cancer remnants were not detected in all four cases. In case \#5, radical cesarean hysterectomy was performed at 31 gestational weeks. After cesarean delivery, two patients (cases \#1 and \#5) received adjuvant chemotherapy with paclitaxel and carboplatin because of deep stromal invasion (case \#1) or the involvement of lymphatic space of cancer cells (case \#5). Case \#6, who received abdominal radical hysterectomy at 16 gestational weeks, received adjuvant concurrent chemo-radiotherapy with weekly cisplatin. After treatment for cervical cancer, all seven patients have had no recurrence within the followup period ( $2.4-5.2$ years), and their children have developed appropriately.

\section{Discussion}

In this report, we described the usefulness of PET/MRI for the preoperative evaluation of cervical cancer metastasis during pregnancy. The guidelines for the management of cervical cancer patients from the European Society of Gynaecological Oncology/European Society for Radiotherapy and Oncology/European Society of Pathology recommend MRI and expert ultrasound examination followed by histological verification of cervical cancer involvement of suspicious lymph nodes to evaluate the cancer progression in pregnant patients ${ }^{17}$. Histological verification of nodes is an invasive procedure for pregnant patients, thus we consider that PET/MRI is more advantageous and can be used instead of this procedure.

Recently, it has been reported that FDG-PET imaging combined with CT or MRI are important tools to detect the spread of many cancers. Some groups have reported the efficacy of FDG-PET examination for cervical cancer ${ }^{16,18-20}$. In a meta-analysis, the sensitivity and specificity of PET for lymph node metastases were $74.7 \%(95 \% \mathrm{Cl} 63.3-84.0)$ and $97.6 \%$ (95\% Cl 95.4-98.9), respectively. In this report, the sensitivity and specificity of MRI were reported to be 55.5\% (95\% Cl 49.2-61.7) and 93.2\% (95\% Cl 91.494.0), respectively and those of CT were $57.5 \%$ (95\% Cl 53.5-61.4), and 92.3\% (95\% Cl 91.1-93.5), respectively ${ }^{16}$. In another retrospective analysis, the sensitivity, specificity, and false-negative and false-

positive rates of PET/CT for detecting involved nodes were $53 \%, 75 \%, 6 \%$, and $82 \%$, respectively ${ }^{18}$. In a prospective study of 18 gynecological cancer cases including cervical cancer and high-risk endometrial cancer, PET/MRI detected all primary tumors and regional lymph nodes as well as PET/CT. In addition, $\mathrm{PET} / \mathrm{MRI}$ had higher sensitivity for parametrial and bladder invasion than PET/CT ${ }^{19}$. In another prospective study of 26 gynecological cancer cases including seven cervical cancer cases and 12 ovarian cancer cases, PET/MRI and PET/CT could equally detect regional lymph nodes metastasis ${ }^{20}$. In this report, the authors also reported that PET/MRI could detect parametrial invasion and invasion of the 
upper third of vagina more precisely than PET/CT. Based on these reports, PET/MRI is more effective in evaluating cervical cancer progression than other imaging techniques. Additionally, our detection of pelvic lymph node metastasis before surgery in case \#6 using PET/MRI further supports these reports. However, while the sensitivity of PET examination is relatively high as described above, the size detection limits of this examination have not been described. In case \#6, one of two metastatic lymph nodes could not be detected with preoperative PET/MRI, and a tiny primary cervical tumor in case \#7 was also not detectable. False-negative results, due to small tumor size, and false-positive results, due to infection and inflammation, are possible.

The advantage of FDG-PET imaging during pregnancy is its safety for the fetus. In pregnant rhesus monkey models using $18 \mathrm{~F}$-fluorothymidine, higher radionuclide uptake and longer retention in the fetal liver than in the maternal liver were reported ${ }^{21}$. Zanotti-Fregonara et al. calculated the fetal radiation dose for administration of 18F-FDG using mathematic modeling for placental crossover and fetal uptake. They reported that the estimated fetal dose after administered activity of $200 \mathrm{MBq}$ delivered was from $5.2 \mathrm{mSv}$ in early pregnancy to $1.4 \mathrm{mSv}$ in late pregnancy; these doses were below the threshold for deterministic effects $^{22}$. As a result, this study encouraged the use of FDG-PET examination during pregnancy. In our eight examinations of seven cases, there were no abnormal accumulations in the fetal body and no abnormalities of the children were detected during the follow-up period.

There are unresolved clinical problems related to FDG-PET imaging in pregnancy, especially with regard to the number of times that PET/MRI can be performed in pregnancy. In case \#5, we performed PET/MRI twice in pregnancy, at $18+3$ and $24+2$ gestational weeks, to monitor the cancer progression. Although a single PET examination is tolerable for the fetus, as described above ${ }^{23}$, the safety of repeated PET examinations has not been reported in detail.

\section{Conclusion}

Although we had a limited number of cases with a short follow-up period, we found that FDG-PET imaging is a useful examination tool for the evaluation of cervical cancer before surgery, and is relatively safe for pregnant women and their babies. However, a large-scale study is needed to resolve the above concerns.

\section{Abbreviations}

FDG: Fluorodeoxyglucose, PET: positron emission tomography, MRI: Magnetic resonance imaging, SUV: standardized uptake value

\section{Declarations}

Ethics approval and consent to participate: The study was approved by the ethics committee of Niigata University (approval No. 2020-0232). Written informed consent was obtained from all patients. 
Consent for publication: The authors obtained written informed consent to publish the clinical information and any accompanying images from the patient.

Availability of data and material: Of the seven cases presented in this study, three cases (cases \#1-\#3) have been previously published ${ }^{8}$. Other all data analyzed during this study are included in this report.

Competing interests: The authors report no conflicts of interest.

Funding: There is no source of financial support or funding.

\section{Authors' contributions:}

Conception and design, T.I., N.N., and T.E.

Writing, review, and/or revision of the manuscript, T.I., S.I., and T.E.

Collect the clinical data, T.I., N.N., S.I., K.Y., K.H., M.Y., S.A., T.W., and S.S.

Study supervision, T.E.

All authors have read and approved the manuscript.

\section{Acknowledgements:}

The authors wish to thank Eiri Shima, Mina Itsukaichi, Koji Nishino, and Masayuki Sekine for their clinical support. They report no conflicts of interest.

Disclosure statement: The authors report no conflicts of interest.

\section{References}

1. Cohen PA, Jhingran A, Oaknin A, Denny L. Cervical cancer. Lancet 2019;393:169-82.

2. Herfs M, Yamamoto Y, Laury A, Wang X, Nucci MR, McLaughlin-Drubin ME, et al. A discrete population of squamocolumnar junction cells implicated in the pathogenesis of cervical cancer. Proc Natl Acad Sci USA 2012;109:10516-21.

3. Hanley SJ, Yoshioka E, Ito Y, Kishi R. HPV vaccination crisis in Japan. Lancet 2015;385:2571.

4. Ikeda S, Ueda Y, Yagi A, Matsuzaki S, Kobayashi E, Kimura T, et al. HPV vaccination in Japan: what is happening in Japan? Expert Rev Vaccines 2019;18:323-5.

5. Utada M, Chernyavskiy P, Lee WJ, Franceschi S, Sauvaget C, de Gonzalez AB, et al. Increasing risk of uterine cervical cancer among young Japanese women: Comparison of incidence trends in Japan, South Korea and Japanese-Americans between 1985 and 2012. Int J cancer 2019;144:2144-52.

6. Kobayashi Y, Tabata T, Omori M, Kondo E, Hirata T, Yoshida K, et al. A Japanese survey of malignant disease in pregnancy. Int J Clin Oncol 2019;24:328-33. 
7. Morice P, Uzan C, Gouy S, Verschraegen C, Haie-Meder C. Gynaecological cancers in pregnancy. Lancet 2012;379:558-69.

8. Yoshihara K, Ishiguro T, Chihara M, Shima E, Adachi S, Isobe M, et al. The Safety and Effectiveness of Abdominal Radical Trachelectomy for Early-Stage Cervical Cancer During Pregnancy. Int J Gynecol Cancer 2018;28:782-7.

9. Monk BJ, Wang J, Im S, Stock RJ, Peters WA, 3rd, Liu PY, et al. Rethinking the use of radiation and chemotherapy after radical hysterectomy: a clinical-pathologic analysis of a Gynecologic Oncology Group/Southwest Oncology Group/Radiation Therapy Oncology Group trial. Gynecol Oncol 2005;96:721-8.

10. Zhou J, Ran J, He ZY, Quan S, Chen QH, Wu SG, et al. Tailoring Pelvic Lymphadenectomy for Patients with Stage IA2, IB1, and IIA1 Uterine Cervical Cancer. J Cancer 2015;6:377-81.

11. Lee KB, Lee JM, Park CY, Lee KB, Cho HY, Ha SY. Lymph node metastasis and lymph vascular space invasion in microinvasive squamous cell carcinoma of the uterine cervix. Int $\mathrm{J}$ Gynecol Cancer 2006;16:1184-7.

12. van Meurs $H$, Visser O, Buist MR, Ten Kate FJ, van der Velden J. Frequency of pelvic lymph node metastases and parametrial involvement in stage IA2 cervical cancer: a population-based study and literature review. Int J Gynecol Cancer 2009;19:21-6.

13. Gien LT, Covens A. Lymph node assessment in cervical cancer: prognostic and therapeutic implications. J Surg Oncol 2009;99:242-7.

14. Bhatla N, Aoki D, Sharma DN, Sankaranarayanan R. Cancer of the cervix uteri. Int J Gynecol Obstet 2018;143:22-36.

15. Schuler MK, Platzek I, Beuthien-Baumann B, Fenchel M, Ehninger G, van den Hoff J. (18)F-FDG $\mathrm{PET} / \mathrm{MRI}$ for therapy response assessment in sarcoma: comparison of PET and MR imaging results. Clin Imaging 2015;39:866-70.

16. Selman TJ, Mann C, Zamora J, Appleyard TL, Khan K. Diagnostic accuracy of tests for lymph node status in primary cervical cancer: a systematic review and meta-analysis. CMAJ 2008;178:855-62.

17. Cibula D, Pötter R, Planchamp F, Avall-Lundqvist E, Fischerova D, Haie Meder C, et al. The European Society of Gynaecological Oncology/European Society for Radiotherapy and Oncology/European Society of Pathology Guidelines for the Management of Patients With Cervical Cancer. Int J Gynecol Cancer 2018;28:641-55.

18. Brunette LL, Bonyadlou S, Ji L, Groshen S, Shuster D, Mehta A, et al. Predictive Value of FDG PET/CT to Detect Lymph Node Metastases in Cervical Cancer. Clin Nucl Med 2018;43:793-801.

19. Schwartz M, Gavane SC, Bou-Ayache J, Kolev V, Zakashansky K, Prasad-Hayes M, et al. Feasibility and diagnostic performance of hybrid PET/MRI compared with PET/CT for gynecological malignancies: a prospective pilot study. Abdom Radiol (New York) 2018;43:3462-7.

20. Queiroz MA, Kubik-Huch RA, Hauser N, Freiwald-Chilla B, von Schulthess G, Froehlich JM, et al. $\mathrm{PET} / \mathrm{MRI}$ and PET/CT in advanced gynaecological tumours: initial experience and comparison. Eur Radiol 2015;25:2222-30. 
21. Bartlett RM, Nickles RJ, Barnhart TE, Christian BT, Holden JE, DeJesus OT. Fetal dose estimates for (18)F-fluoro-L-thymidine using a pregnant monkey model. J Nucl Med 2010;51:288-92.

22. Brent RL. Saving lives and changing family histories: appropriate counseling of pregnant women and men and women of reproductive age, concerning the risk of diagnostic radiation exposures during and before pregnancy. Am J Obstet Gynecol 2009;200:4-24.

23. Zanotti-Fregonara P, Stabin MG. New Fetal Radiation Doses for (18)F-FDG Based on Human Data. J Nucl Med 2017;58:1865-6.

\section{Tables}

Due to technical limitations, table 1 is only available as a download in the Supplemental Files section.

\section{Figures}
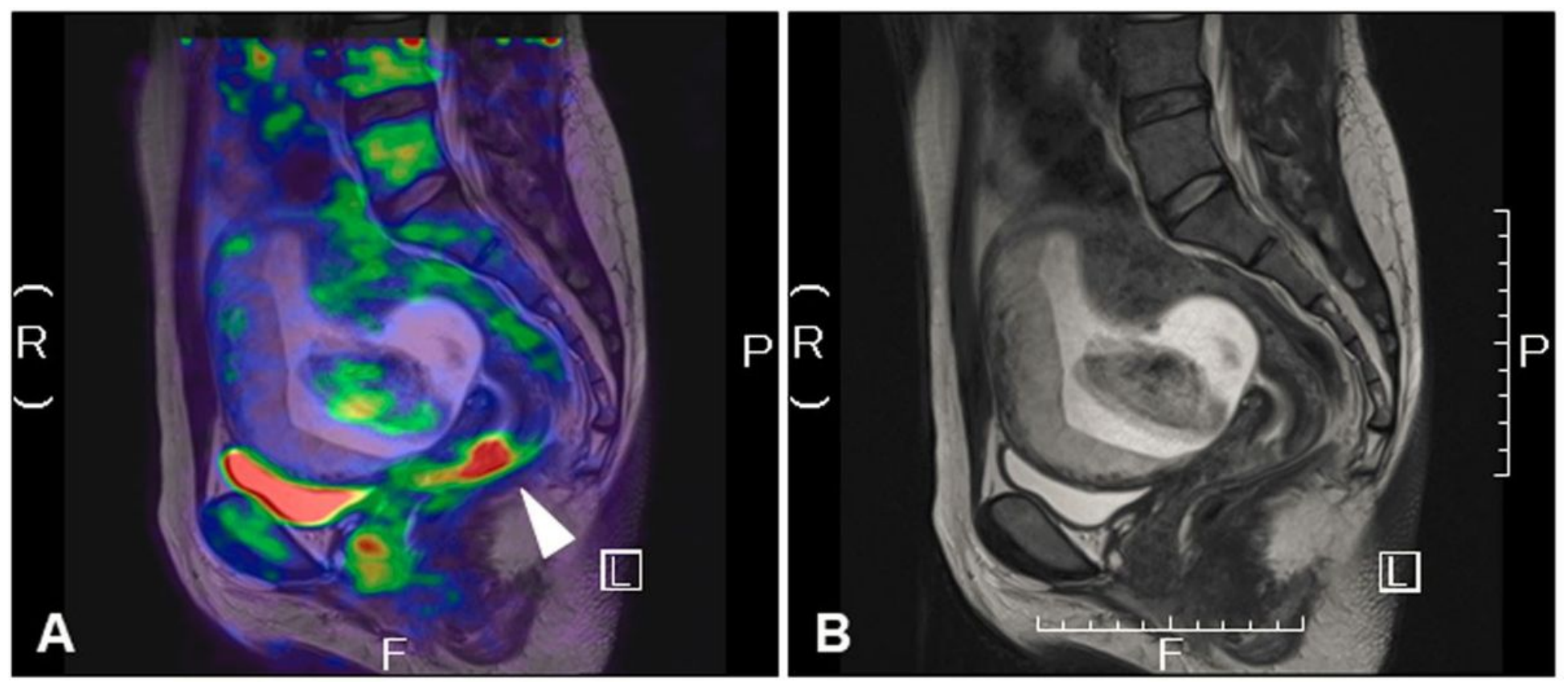

Figure 1

PET/MR images of case \#3 (A) Sagittal view of PET/MR fusion image shows increased FDG uptake of cervical tumor (white arrowhead). (B) T2-weighted magnetic resonance image corresponding to PET/MR image $(A)$ 

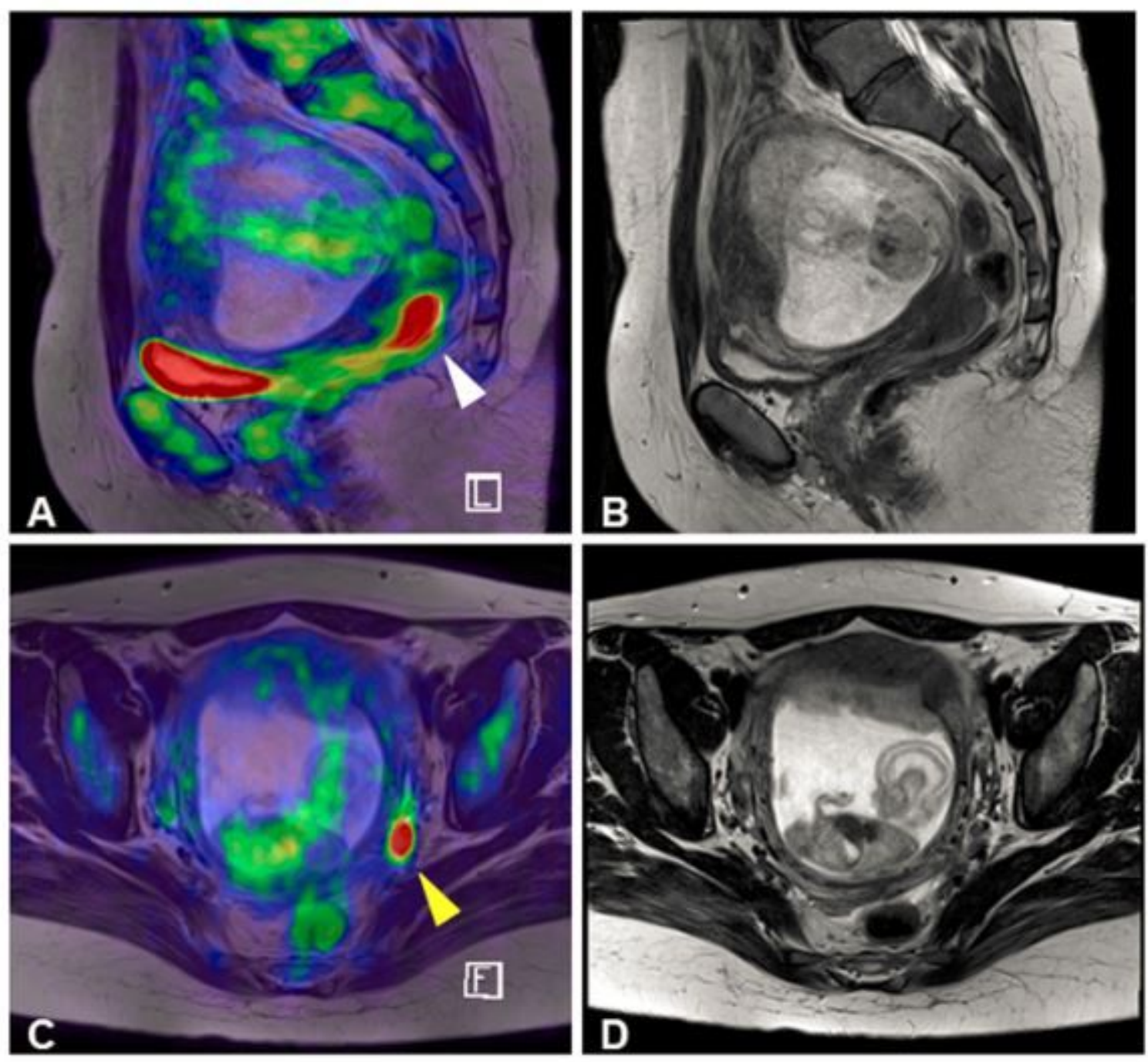

Figure 2

PET/MR images of case \#6 (A) Sagittal views of PET/MR fusion image show increased FDG uptake of cervical tumor (white arrowhead). (B) T2-weighted magnetic resonance image corresponding to PET/MR image (A) (C) Axial views of PET/MR fusion image show increased FDG uptake of pelvic lymph node (yellow arrowhead). (D) T2-weighted magnetic resonance image corresponding to PET/MR image (C)

\section{Supplementary Files}

This is a list of supplementary files associated with this preprint. Click to download.

- PETMRItableBMC2.xIsx 